\title{
Energy stored during high pressure torsion of pure metals
}

\author{
A.P. Zhilyaev \\ alexz@anrb.ru \\ Nosov Magnitogorsk State Technical University, 38 Lenin St., Magnitogorsk, 455000, Russia \\ Institute for Metals Superplasticity Problems, RAS, 39 Khalturin St., Ufa, 450001, Russia
}

In the paper a problem of estimating the energy released during plastic deformation of pure metals by the high-pressure torsion (HPT) method is discussed. The work accomplished during plastic deformation by torsion under high pressure implies considerable heating of the sample. However, measurements using thermocouples located near the deformation zone show a temperature rise in the range of 5-50 degrees. As an example, pure nickel was chosen, for which there are experimental data on the kinetics of the formation of vacancies and dislocations in the process of torsion under high pressure and data on the refinement of the microstructure in the HPT process. It has been established that the amount of heat expended in heating the massive anvils would be sufficient to evaporate the nickel disc. The energy of formation of crystal structure defects (vacancies, dislocations, and high-angle grain boundaries) in nickel subjected to high-pressure torsion is also estimated. The correct equation for calculating the magnitude of the equivalent strain at simple shear is presented. It is shown that this calculation leads to a good agreement between the values of plastic energy and the work expended on the formation of the defective structure and heating of the sample-anvil system. Using the von Mises equation for calculations leads to the value of the work done during plastic deformation by torsion, which is two orders of magnitude higher than the upper estimate of the energy spent on heating the anvil and the formation of defects (vacancies, dislocations and new grain boundaries) of the crystal lattice. An asymptotic equation is proposed for calculating the value of equivalent strains for small $(<1)$ and large $(>2)$ shear strain degrees.

Keywords: severe plastic deformation, nickel, released heat, equivalent strain.

УДК: $548.4+538.91$

\section{Энергия, выделяемая в чистых металлах при деформации кручением под высоким давлением}

\author{
Жиляев А.П. \\ alexz@anrb.ru \\ Магнитогорский государственный технический университет им. Г. И. Носова, пр. Ленина, 38, Магнитогорск, \\ 455000, Россия \\ Институт проблем сверхпластичности металлов РАН, ул. Халтурина, 39, Уфа, 450001, Россия
}

В работе обсуждается проблема оценки энергии, выделяемой при пластической деформации чистых металлов методом кручения под высоким давлением (КВД). Работа, совершенная при пластической деформации кручением под высоким давлением, предполагает значительный разогрев образца. Однако, измерения с помощью термопар, расположенных вблизи зоны деформации, показывают повышение температуры в пределах 5 - 50 градусов. В качестве примера для анализа выбран чистый никель, для которого имеются экспериментальные данные по кинетике образования вакансий и дислокаций и измельчению микроструктуры в процессе кручения под высоким давлением. Установлено, что количества теплоты, затраченной на разогрев массивных наковален, было бы достаточно для испарения образца никеля в виде диска. Также оценена энергия образования дефектов кристаллической структуры (вакансий, дислокаций и большеугловых границ зерен) в никеле, подвергнутом деформации кручением под высоким давлением. Представлено корректное уравнение для расчета величины эквивалентной деформации при простом сдвиге. Показано, что расчет по этому уравнению приводит к хорошему согласию значений пластической энергии и работы, затраченной на формирование дефектной структуры и нагрев системы образец-наковальни. Использова- 
ние уравнения фон Мизеса для расчетов приводит к значению работы, совершенной в ходе пластической деформации кручением, на два порядка превышающему верхнюю оценку величины энергии, затраченной на нагрев наковален и образование дефектов (вакансий, дислокаций и новых границ зерен) кристаллической решетки. Предложено асимптотическое уравнение для расчета значения эквивалентной деформаций для малых $(e<1)$ и больших $(e>2)$ степеней сдвиговой деформации.

Ключевые слова: интенсивная пластическая деформация, никель, выделяемая теплота, эквивалентная деформация.

\section{1. Введение}

Интенсивная пластическая деформация (ИПД) является эффективным методом измельчения зерна в металлических сплавах $[1,2]$. Среди многочисленных процессов ИПД для получения объемных наноструктурных материалов наиболее часто используются равноканальное угловое прессование (РКУП) [3] и кручение под высоким давлением (КВД) [4]. Первоначально считалось, что процессы ИПД протекают при температуре окружающей среды, которая соответствует низкой гомологической температуре, обычно $T<0.3 \cdot T_{M}$, для большинства металлических материалов, таких как $\mathrm{Cu}$ [5], Ni [6,7], Ti [8], Zr [9] и др.

Из указанных двух методов ИПД, наиболее эффективным способом измельчения структуры является кручение под высоким давлением [10]. Предполагалось, что при низких скоростях деформации в дисках, деформируемых КВД, могут преобладать приблизительно изотермические условия, поскольку наковальни имеют хорошую теплопроводность, и начальная температура образцов не будет сильно увеличиваться. Однако повышение температуры в процессе ИПД было обнаружено для нескольких металлов. Ямагути и соавт. [11] использовали термопары, встроенные в заготовки, и зафиксировали повышение температуры на $25-30^{\circ} \mathrm{C}$ за время одного прохода РКУП для первоначально отожженного чистого алюминия. Жиляев и др. [12] на основе косвенной оценки по эволюции растворения и выпадения вторичной фазы показали повышение температуры на $\sim 110-140^{\circ} \mathrm{C}$ во время КВД литого сплава Al-7 мас.\% Si. Тодака и соавт. [13] использовали термопару, встроенную в верхнюю наковальню КВД, и зафиксировали изменение температуры от 20 до $\sim 45^{\circ} \mathrm{C}$ в месте крепления термопары, которая была примерно на 1 мм выше верхней поверхности образцов алюминия высокой чистоты. Алюминий, медь, железо и молибден были впоследствии выбраны в качестве модельных материалов в работе [14], в которой было установлено, что температура увеличивается на ранних стадиях деформации, но насыщается до устойчивых уровней при больших деформациях. Было обнаружено увеличение температуры на $5^{\circ} \mathrm{C}$ для алюминия, на $15^{\circ} \mathrm{C}$ для меди и на $25^{\circ} \mathrm{C}$ для железа (все металлы деформировались при $P=2$ ГПа со скоростью 1 об/мин). Было установлено, что повышение температуры пропорционально твердости и скорости вращения. Недавно в работе [15] путем внедрения термопары на край образца было измерено непосредственно изменение температуры в процессе КВД. Прирост температуры на $10-12^{\circ} \mathrm{C}$ был зафиксирован для чистой меди и титана примерно за 30 секунд деформации (давление 2 ГПа), что соответствует половине оборота для скорости вращения 1 об/мин. Данные результаты в целом согласуются с данными непрямых измерений [16]. Настоящая работа представляет собой попытку оценить количество теплоты, выделенной в процессе интенсивной пластической деформации образцов из чистых металлов, и оценить работу, затраченную на создание дефектов кристаллической решетки (вакансий, дислокаций и границ зерен). В качестве примера был выбран чистый никель, для которого имеются экспериментальные данные по кинетике образования вакансий и дислокаций в процессе кручения под высоким давлением [17].

\section{2. Оценка пластической работы, выделяющейся в процессе КВД}

Как уже отмечалось выше, в качестве материала был выбран чистый никель, в качестве материала бойков обычно используют инструментальную сталь P6M5 (H13). Физические характеристики материала бойков и никеля представлены в Табл. 1. В процессе кручения энергия, которая выделяется при пластической деформации, идет на разогрев системы и создание новых дефектов, таких как вакансии, дислокации и большеугловые границы зерен.

Оценим количество теплоты, необходимое для нагрева двух бойков общей массой 0.9 кг при КВД никеля. В качестве оценки прироста температуры возьмем данные из работы [16], приведенные на Рис. 1. Необходимое для нагрева бойков количество теплоты на единицу объема вычисляется по формуле:

$$
Q=Q_{\text {HЕАT }} / V=\rho C_{p} \Delta T=\left\{\begin{array}{c}
89.7 \frac{\text { МДж }}{\mathrm{m}^{3}}, \text { для } N=6 \\
125.6 \frac{\mathrm{MД}}{\mathrm{m}^{3}}, \text { для } N=10
\end{array}\right.
$$

Для бойков массой около одного килограмма потребуется 10.35 мДж (для $\Delta T=25^{\circ} \mathrm{C}, N=6$ ) или 14.49 мДж (для $\Delta T=35^{\circ} \mathrm{C}, N=10$ ). Кажущееся небольшим количество теплоты, выделяемое в процессе КВД, оказывается значительным, если учитывать малые размеры дисков образцов. Данное количество теплоты, переданное на диск никеля, испарило бы его. Однако, этого не происходит из-за хорошей теплопроводности материалов (стали и никеля) находящимися в плотном контакте и, таким образом, бойки служат практически неограниченным стоком для тепловой энергии, генерируемой в процессе КВД.

Работа, затраченная на создание вакансий, может быть оценена по максимальной концентрации вакансий, достигнутой в процессе кручения, которая составляет $10^{-5}[17]$. Тогда энергия на единицу объема, затраченная на достижение такой концентрации вакансий, равна

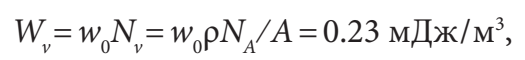


где $w_{0} \sim 1.5 \mathrm{eV}=2.4 \cdot 10^{-19}$ Дж - энергия образования одной вакансии в никеле, $N_{A}=6.022 \cdot 10^{23} 1 /$ моль - число Авогадро, $A=56.7$ кг/кмоль - молярный вес никеля и $\rho$ - его плотность.

Известно, что КВД приводит к высокой накопленной плотности дислокаций, которая может достигать значений $8 \cdot 10^{15} \mathrm{M}^{-2}$ [17]. Данная величина хорошо согласуется с оценками плотности дислокаций в никеле после КВД, полученными в работах других авторов (см., например, обзор [4]). Работу, затраченную на создание такой плотности дислокаций в никеле, можно оценить как

$$
W_{d}=\rho_{d} w_{0 d} \approx \rho_{d} \frac{\mu b^{2}}{4 \pi} \ln \left(\frac{R_{C}}{r}\right) \sim 16.5 \text { мДж/ } \mathrm{M}^{3},
$$

где $\rho_{d}$ - максимальная плотность дислокаций в КВД никеля, $w_{0 d}-$ энергия ядра краевой дислокации, $R_{C}-$ paдиус обрезания и может быть принят равным размеру зерна ( 100 нм), а размер ядра дислокации принимается равным двум векторам Бюргерса, $2 b=4.98 \cdot 10^{-9}$ нм.
Энергию, затраченную на создание высокой удельной плотности большеугловых границ зерен, или, другими словами, на измельчение микроструктуры, можно оценить по формуле

$$
W_{G B}=\gamma_{G B} \frac{S}{V} \approx \frac{6 \gamma_{G B}}{d} \sim 60 \mathrm{м} Д ж / \mathrm{m}^{3},
$$

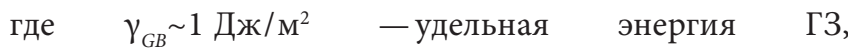
a $d \sim 100$ мн - средний размер зерна.

Следует отметить, что оценки, проведенные по формулам (2)-(4), это максимальные значения энергий, полученных для максимальных плотностей дефектов кристаллической решетки, создаваемых в процессе интенсивной пластической деформации.

Суммируя значения энергии, затраченной на тепловой разогрев (1) и на создание дефектов (2)-(4), можно оценить сверху величину работы пластической деформации (для $N=6$ ) как

$$
W_{T}=Q+W_{V}+W_{d}+W_{G B} \sim 89.7+76.7=166.4 \mathrm{м} Д ж / \mathrm{m}^{3}
$$

\begin{tabular}{|c|c|c|c|c|c|c|}
\hline Материал & $\begin{array}{c}\text { Плотность } \\
\rho, 10^{3}\left(\text { кг/ }{ }^{3}\right)\end{array}$ & $\begin{array}{c}\text { Теплоемкость } \\
C_{p}, 10^{3}\left(\text { Дж/кг }{ }^{\circ} \mathrm{C}\right)\end{array}$ & $\begin{array}{c}\text { Теплопроводность } \\
k,\left(\mathrm{BT} / \mathrm{M}^{\circ} \mathrm{C}\right)\end{array}$ & $\begin{array}{c}\text { Температуро- } \\
\text { проводность } \\
\alpha=k /\left(\rho \cdot C_{p}\right), 10^{-6}\left(\mathrm{M}^{2} / \mathrm{c}\right)\end{array}$ & $\begin{array}{l}\text { Bec } \\
(\kappa г)\end{array}$ & Комментарии \\
\hline Material & $\begin{array}{c}\text { Density } \\
\rho, 10^{3}\left(\mathrm{~kg} / \mathrm{m}^{3}\right)\end{array}$ & $\begin{array}{c}\text { Heat capacity } \\
C_{P}, 10^{3}\left(\mathrm{~J} / \mathrm{kg}^{\circ} \mathrm{C}\right)\end{array}$ & $\begin{array}{l}\text { Thermo- } \\
\text { conductivity } \\
k,\left(\mathrm{~W} / \mathrm{m}^{\circ} \mathrm{C}\right)\end{array}$ & $\begin{array}{c}\text { Temperature } \\
\text { conductivity } \\
\alpha=k /\left(\rho \cdot C_{P}\right), 10^{-6}\left(\mathrm{~m}^{2} / \mathrm{s}\right)\end{array}$ & $\begin{array}{l}\text { Weight } \\
(\mathrm{kg})\end{array}$ & Comments \\
\hline $\begin{array}{c}\text { Сталь/ } \\
\text { Steel H13 }\end{array}$ & 7800 & 0.46 & 25.0 & 6.97 & $2 \times 0.450$ & $\begin{array}{l}\text { Бойки/ } \\
\text { Anvils }\end{array}$ \\
\hline $\begin{array}{c}\text { Никель/ } \\
\text { Nickel }\end{array}$ & 8908 & 0.44 & 90.9 & 23.00 & 0.0007 & $\begin{array}{c}\text { КВД диск/ } \\
\text { НРТ disk }\end{array}$ \\
\hline
\end{tabular}

Табл. 1. Физические свойства никеля и инструментальной стали. Table 1. Physical properties of nickel and steel H13.

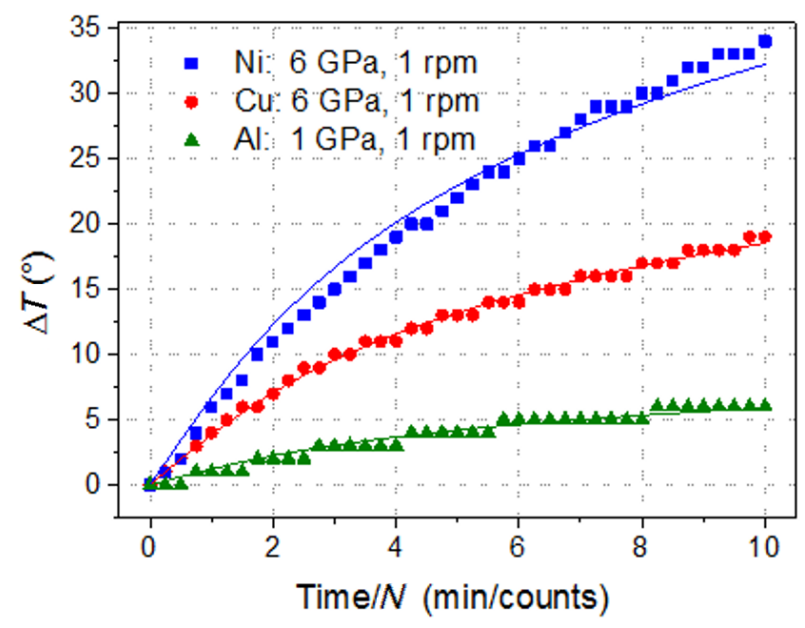

a

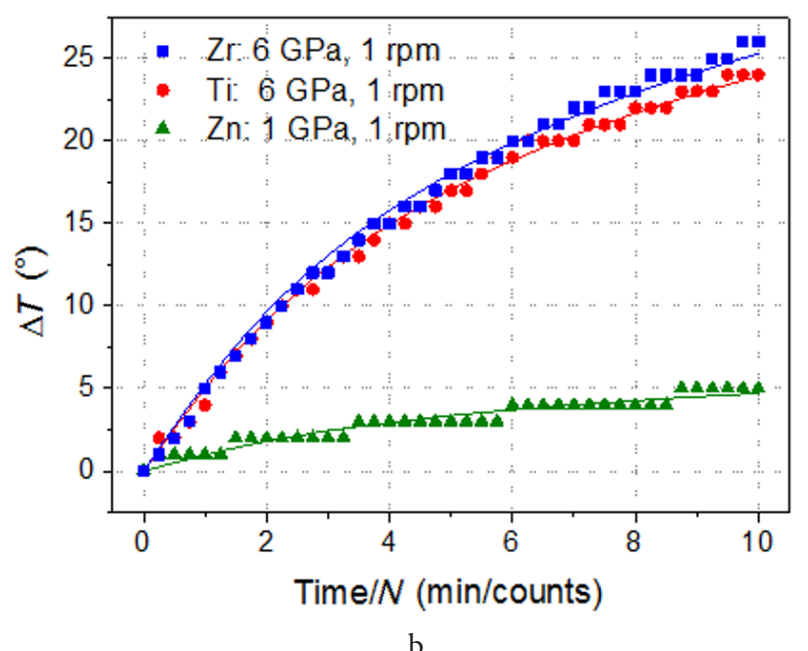

$\mathrm{b}$

Рис. 1. Прирост температуры в зависимости от времени (числа оборотов) КВД деформации для ГЦК (Al, $\mathrm{Cu}, \mathrm{Ni})(\mathrm{a})$ и ГПУ (Zn, Ti, $\mathrm{Zr}$ ) металлов (b). Условия деформации указаны на рисунке [16].

Fig. 1. Temperature increase as a function of time (number of turns) for fcc (Al, Cu, Ni) (a) and hcp ( $\mathrm{Zn}, \mathrm{Ti}, \mathrm{Zr}$ ) metals (b) subjected to HPT. Processing conditions are shown in inset [16]. 
Величину работы, совершенной по время пластической деформации, можно оценить, зная механические свойства наноструктурного никеля, полученного КВД.

$$
W_{H P T}=\tau \cdot \gamma \approx \frac{H_{v}}{6} \frac{2 \pi N r}{h}
$$

где $\tau-$ сдвиговое напряжение никеля, которое можно оценить, зная микротвердость [18], а $\gamma$ - накопленная сдвиговая деформация, определенная исходя из геометрии КВД. Оценка пластической энергии по формуле (6) для $N=6$ полных оборотов дает величину около 15000 мДж/ м $^{3}$, что на два порядка выше величины верхней оценки суммы энергии дефектной структуры никеля и выделившегося тепла в системе диск-наковальни. Кроме того, следует отметить, что энергия, вычисляемая по формуле (6), линейно зависит от числа оборотов $(N)$. В то время как экспериментально установлено, что микроструктурные характеристики (микротвердость, средний размер зерна, плотность дислокаций и др.) имеют тенденцию к насыщению, т.е. мало изменяются с увеличением накопленной деформации после некоторого критического значения. Для никеля такое критическое значение для микротвердости является число полных оборотов $N$ равное пяти. Такую же тенденцию обнаруживает и прирост температуры, показанный на Рис. 1. С ростом накопленной деформации наблюдается снижение прироста температуры, хотя данный факт может быть связан с увеличением теплового рассеяния с поверхности бойков. Уравнение (6) в левой своей части корректно отражает тот факт, что величина работы пластической деформации не должна зависеть от пути деформации и должна быть равна площади под кривой зависимости $\tau(\gamma)$. А вот вычисление накопленной деформации в зависимости от угла поворота (или количества полных оборотов) должно быть скорректировано. Как показали в своих работах Онака [19] и Стюве [20], при деформации простым сдвигом (а кручение под высоким давлением как раз и есть такой случай) направление и величину максимального сдвига можно найти из простого геометрического рассмотрения (Рис. 2). При простом сдвиге на величину $\gamma=r \cdot \varphi / h$ элементарный стержень длиной $l_{0}$ превращается в стержень длиной $l$ (Рис. 2). Тогда, находя из теоремы Пифагора, получим начальную величину $l_{0}^{2}=1+x^{2}$ и конечную величину $l^{2}=1+(\gamma+x)^{2}$ как функцию $x$ и величины сдви-

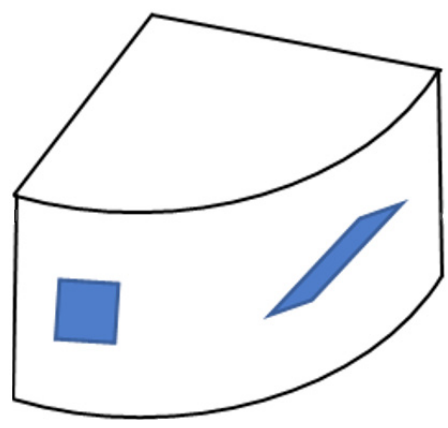

a говой деформации. Максимальное удлинение стержня можно найти, максимизируя функцию $\frac{d}{d x} \frac{l}{l_{0}}=0$. Тогда положительный корень данного уравнения равен

$$
x=-\frac{\gamma}{2}+\sqrt{1+\frac{\gamma^{2}}{4}}
$$

Подставляя значение $x$ в уравнение $\varepsilon_{e q}=\ln \left(l / l_{0}\right)_{\text {мАХ, }}$ эквивалентная деформация может быть вычислена по формуле

$$
\varepsilon_{e q}=\ln \left(\frac{l}{l_{0}}\right)_{M A X}=\frac{1}{2} \frac{1+\left(\frac{\gamma}{2}+\sqrt{1+\frac{\gamma^{2}}{4}}\right)^{2}}{1+\left(-\frac{\gamma}{2}+\sqrt{1+\frac{\gamma^{2}}{4}}\right)^{2}}
$$

Для фиксированного отношения радиуса диска к его толщине $r / h=5$, соответствующего середине радиуса диска, зависимость эквивалентной деформации от угла поворота $\varphi$ представлена на Рис. 3. Очевидно, что для значений $\varphi>2$, зависимость (8) может быть аппроксимирована функцией $\ln (r \varphi / h)$, а при малых углах кручения хорошо работает линейная аппроксимация и выполняется приближение von Mises (Рис. 3). С учетом уравнения (8) вычисление работы, совершаемой при интенсивной пластической деформации, дает значение $\mathrm{W}_{\text {нРт }} \sim 150$ МДж/м³. Данное значение хорошо коррелирует со значением, полученным из уравнения (5).

\section{3. Краткие выводы}

В работе установлено, что энергии, выделяющейся в процессе пластической деформации кручением под высоким давлением, достаточно не только для генерации высокодефектной микроструктуры никеля, но и для существенного термического разогрева наковален. Установлено, что количества теплоты, затраченной на разогрев массивных наковален, было бы достаточно для испарения образца никеля в виде диска. Также оценена энергия образования дефектов кристаллической структуры (вакансий, дислокаций и большеугловых границ зерен) в никеле, подвергнутом деформации кручением под высоким давлением. Представлено корректное уравнение для расчета величины эквивалентной деформации при простом сдвиге. Показано, что расчет по этому уравнению приводит к хорошему согласию

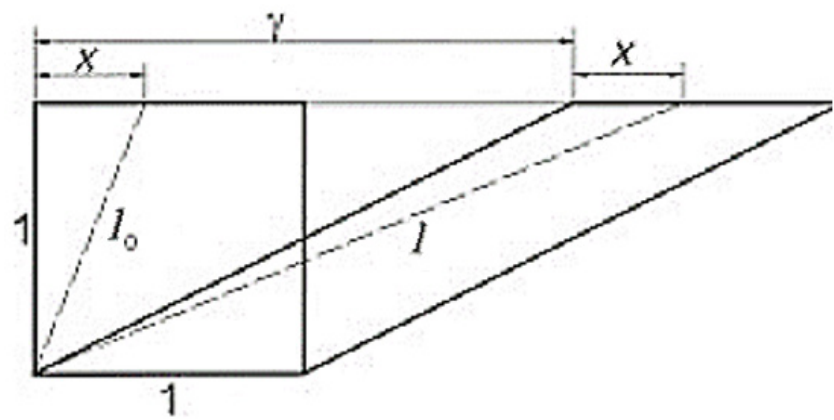

b

Рис. 2. Схема деформации элементарной ячейки при деформации кручением (а) и максимальное удлинение при деформации простым сдвигом (b).

Fig. 2. Schematic of skewing elemental square (a) and maximum elongation in simple shear (b). 


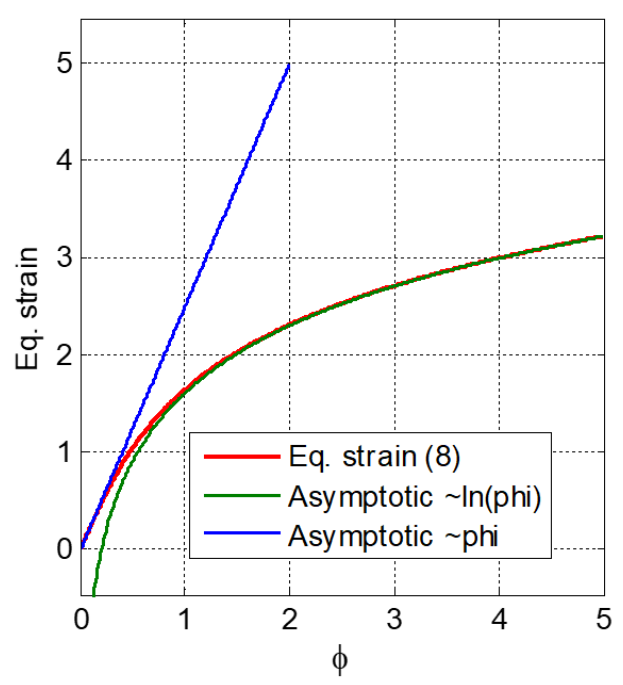

Puc. 3. (Color online) Зависимость величины эквивалентной степени деформации для случая простого сдвига как функции угла поворота для КВД. Для сравнения приведены также асимптотики для малых и больших углов поворота.

Fig. 3. (Color online) Equivalent strain as a function of number of whole revolutions, $N$. Asymptotic for small and large rotation angle of HPT are also shown.

значений пластической энергии и работы, затраченной на формирование дефектной структуры и нагрев системы образец-наковальни. Использование уравнения фон Мизеса для расчетов приводит к значению работы, совершенной в ходе пластической деформации кручением, на два порядка превышающему верхнюю оценку величины энергии, затраченной на нагрев наковален и образование дефектов (вакансий, дислокаций и новых границ зерен) кристаллической решетки. Предложено асимптотическое уравнение для расчета значения эквивалентной деформаций для малых $(e<1)$ и больших $(e>2)$ степеней сдвиговой деформации.

Благодарности/Acknowledgements. Работа выполнена при финансовой поддержке Министерства науки и Высшего образования Российской Федерации (Грант 14. Z50.31.0043)./Author acknowledges the financial support of Ministry for Science and Higher Education of Russian government (Grant 14. Z50.31.0043).

\section{Литература/References}

1. R.Z. Valiev, A.V. Korznikov, R.R. Mulyukov. Mater. Sci.Eng. A. 168, 141 (1993). DOI: 10.1016/0921-5093(93)90717-S

2. R.Z. Valiev, A.P. Zhilyaev, T.G. Langdon. Bulk nanostructured materials: Fundamentals and applications. New Jersey, Wiley \& Sons. (2014) 450 p.

3. R.Z. Valiev, T.G. Langdon. Progr. Mater. Sci. 51, 881 (2006). DOI: 10.1016/j.pmatsci.2006.02.003

4. A.P. Zhilyaev, T. G. Langdon. Progr. Mater. Sci. 53, 893 (2008). DOI: 10.1016/j.pmatsci.2008.03.002

5. A. Gimazov, A. Zhilyaev. Let. Mater. 6, 237 (2016). (in Russian) [А.А. Гимазов, А.П. Жиляев. Письма о материалах. 6, 237 (2016).] DOI: 10.22226/2410-3535-2016-3-231-236
6. A. P. Zhilyaev, G. V. Nurislamova, B-K. Kim, M.D. Baró, J. A. Szpunar, T. G. Langdon. Acta Mater. 51, 753 (2003). DOI: 10.1016/S1359-6454(02)00466-4

7. A.A. Mukhametgalina, A.A. Samigullina, S.N. Sergeyev, A.P. Zhilyaev, A.A. Nazarov, Y.R. Zagidullina, N.Y. Parkhimovich, V.V. Rubanik, Y.V. Tsarenko. Let. Mater. 7, 85 (2017). (in Russian) [А.А. Мухаметгалина, А.А. Самигуллина, С.Н. Сергеев, А.П. Жиляев, А.А. Назаров, Ю. Р. Загидуллина, Н. Ю. Пархимович, В.В. Рубаник, Ю.В. Царенко. Письма о материалах. 7, 85 (2017).] DOI: 10.22226/2410-3535-2017-2-85-90

8. A.P. Zhilyaev, V.A. Popov, A.R. Sharafutdinov, V.N. Danilenko. Let. Mater. 1, 203 (2011).(in Russian) [А.П. Жиляев, В.А. Попов, А.Р. Шарафутдинов, В. Н. Даниленко. Письма о материалах. 1, 203 (2011).] DOI: 10.22226/2410-3535-2011-4-203-207

9. M. T. Perez-Prado, A. Sharafutdinov, A. P. Zhilyaev. Mater. Let. 64, 211 (2010). DOI: 10.1016/j.matlet.2009.10.049

10. R.Z. Valiev, A.P. Zhilyaev, T.G. Langdon. Bulk Nanostructured Materials: Fundamentals and Applications. Saint-Petersburg, Eco-Vector. (2017). 480 p. (in Russian) [Р. 3. Валиев, А. П. Жиляев, Т. Дж. Лэнгдон. Объемные наноструктурные материала: фундаментальные основы и применения. Санкт-Петербург, Эко-вектор (2017) 480 с.]

11. D. Yamaguchi, Z. Horita, M. Nemoto, T.G. Langdon. Scripta Mater. 41, 791 (1999). DOI: 10.1016/S1359-6462(99)00233-X

12. A.P. Zhilyaev, J.M. Garcia-Infanta, F. Carreno, T. G. Langdon, O. A. Ruano. Scripta Mater. 57, 763 (2007). DOI: 10.1016/j.scriptamat.2007.06.029

13. Y. Todaka, M. Umemoto, A. Yamazaki, J. Sasaki, K. Tsuchiya. Mater. Trans. 49, 7 (2008). DOI: 10.2320/matertrans.ME200713

14. K. Edalati, R. Miresmaeili, Z. Horita, H. Kanayama, R. Pippan. Mater. Sci.Eng. A. 528, 7301 (2011). DOI: 10.1016/j.msea.2011.06.031

15. K. Edalati, Yu. Hashiguchi, P. H. R. Pereira, Z. Horita, T.G. Langdon. Mater. Sci.Eng. A. 714, 167 (2018). DOI: 10.1016/j.msea.2017.12.095

16. A.P. Zhilyaev, T.G. Langdon. IOP 2014 IOP Conf. Series: Mater. Sci. Eng. 63, 012052 (1-7) (2014). DOI: $10.1088 / 1757-899 X / 63 / 1 / 012052$

17. E. A. Korznikova. Issledovaniye kontsentratsii vakansiy i plotnosti dislokatsiy v GTSK metallakh posle intensivnoy plasticheskoy deformatsii. Dissertacija na soiskanie stepeni kandidata tehnicheskih nauk. Ufa (2011) 140 p. (in Russian) [Исследование концентрации вакансий и плотности дислокаций в ГЦК металлах после интенсивной пластической деформации: дисс. канд. физ-мат наук. Уфа. 2011. 140 с.]

18. A.P. Zhilyaev, S. Lee, G. V. Nurislamova, R.Z. Valiev, T.G. Langdon. Scripta Mater. 44 (12), 2753 (2001). DOI: 10.1016/S1359-6462(01)00955-1

19. S. Onaka. Phil.Mag. Let. 90633 (2010). DOI: $10.1080 / 09500839.2010 .489030$

20. H.P. Stuwe. Adv Eng. Mater. 5 (3), 291 (2003). DOI: 10.1002 /adem.200310085 